\title{
Treatment strategy and prognostic factors for Krukenberg tumors of gastric origin: report of a 10-year single-center experience from China
}

\author{
Pengfei Yu ${ }^{1}$, Ling Huang ${ }^{1}$, Guoping Cheng ${ }^{2}$, Litao Yang ${ }^{1}$, Gaiguo Dai ${ }^{1}$, Jieer Ying $^{3}$ \\ and Yian Du ${ }^{1}$ \\ ${ }^{1}$ Department of Abdominal Surgery, Zhejiang Cancer Hospital, Hangzhou 310022, China \\ ${ }^{2}$ Department of Pathology, Zhejiang Cancer Hospital, Hangzhou 310022, China \\ ${ }^{3}$ Department of Medical Oncology, Zhejiang Cancer Hospital, Hangzhou 310022, China \\ Correspondence to: Pengfei Yu, email: yupengfei23@163.com \\ Yian Du, email: duyajim@126.com \\ Keywords: Krukenberg tumors, gastric cancer, metastasectomy, chemotherapy, prognosis \\ Abbreviations: OS: overall survival; HIPEC: hyperthermic intraperitoneal chemotherapy; ER: estrogen receptor; PR: progesterone \\ receptor; IHC: immunohistochemistry \\ Received: May 16, $2017 \quad$ Accepted: June 30, $2017 \quad$ Published: August 01, 2017 \\ Copyright: Yu et al. This is an open-access article distributed under the terms of the Creative Commons Attribution License 3.0 (CC \\ BY 3.0), which permits unrestricted use, distribution, and reproduction in any medium, provided the original author and source are \\ credited.
}

\section{ABSTRACT}

Background: Gastric cancer patient with ovarian metastasis is common in clinical practice, but it is still uncertain whether surgical resection of ovarian metastasis could improve the outcome. This study aimed to explore the survival benefit of metastasectomy plus chemotherapy over chemotherapy alone in the treatment of Krukenberg tumors arising from gastric cancer and to identify prognostic factors.

Results: A total of 152 patients were identified, including 93 patients with synchronous ovarian metastasis and 59 patients with metachronous ovarian metastasis. Overall survival (OS) was significantly better in metastasectomy group relative to the non-metastasectomy group for patients with synchronous ovarian metastasis (19.0 months vs. 11.8 months; $P<0.001)$ and those with metachronous ovarian metastasis (24.6 months vs. 14.3 months; $P=0.02)$, respectively. Metastasectomy (hazard ration [HR] 0.486; 95\% confidence interval [CI] 0.323$0.729 ; P<0.001$ ), peritoneal carcinomatosis (HR 1.934; 95\% CI 1.230-3.049; $P=$ 0.004 ), and expression status of ER- $\beta$ (HR 0.404; 95\% CI $0.251-0.648 ; P<0.001$ ) and PR (HR 0.496; 95\% CI 0.301-0.817; P< 0.001) were independent predictors of OS.

Methods: All patients who were diagnosed with gastric cancer and ovarian metastases between January 2005 and December 2014 were included in the current study. Patients were subdivided according to treatment modality: the metastasectomy group (metastasectomy plus chemotherapy) and the non-metastasectomy group (chemotherapy alone). The clinicopathological features and the treatment records were reviewed in detail and their association with survival were analyzed.

Conclusion: Metastasectomy plus chemotherapy was associated with survival benefits in patients with Krukenberg tumors from gastric cancer. Metastasectomy, peritoneal carcinomatosis, and expression status of ER- $\beta$ and PR were independent prognostic factors for survival. 


\section{INTRODUCTION}

Gastric cancer is one of the most common cancers worldwide, and the incidence is particularly high in Asian countries, including China [1]. Metastasis and recurrence are the major causes for poor prognosis in gastric cancer. Ovarian metastasis (Krukenberg tumor) is usually seen in female patients, including synchronous metastasis, and metachronous metastasis after curative resection of gastric cancer [2]. The reported incidence of ovarian metastasis or Krukenberg tumor is approximately $0.3 \%$ to $6.7 \%$, however, some autopsy studies have reported incidence rates of $33 \%$ to $41 \%$ [3, 4]. Ovarian metastasis is associated with poor prognosis, and is one of the most important causes of treatment failure for gastric cancer in female patients [5]. Although systemic chemotherapy can provide symptom palliation and prolonged survival in patients with ovarian metastasis, the efficiency and survival time remain disappointing [2,6]. Several studies have explored the utility of metastasectomy for Krukenberg tumors in patients with gastric cancer [7, 8] However, the role of ovarian metastasectomy is still under debate and is likely to benefit only a specific subset of patients [9]. So far, the optimal treatment strategy for Krukenberg tumors from gastric cancer had not been clearly established. This study was conducted to determine associations between metastasectomy of Krukenberg tumors, clinicopathological features, and survival outcome, and consequently provide optimal treatment strategy for these patients.

\section{RESULTS}

\section{Patient characteristics}

The median follow-up duration for all patients was 37.2 (range 2.5-71) months; median age at treatment onset was 43.4 (range 18-65) years, and mean size of metastatic ovarian tumors was 8.13 (range 2-20) $\mathrm{cm}$.

Clinicopathologic characteristics of gastric cancer with synchronous or metachronous ovarian metastasis are listed in Tables 1 and 2, respectively. Of these, tumor location, differentiation, ascites, ER and PR expression, tumor markers, and TNM stage were similar for both groups of either synchronous or metachronous ovarian metastasis.

\section{Treatment outcome and prognostic factors}

The median OS of patients with synchronous ovarian metastasis was 15.6 months $(95 \%$ confidence interval [CI], 13.9 to 17.3 months). The median OS of metastasectomy group and non-metastasectomy group was 19.0 months (95\% CI,16.6 to 21.5 months) and 11.8 months (95\% CI, 10.1 to 13.5 months), respectively. For patients with metachronous ovarian metastasis, median OS was 21.7 (95\% CI 17.6-25.9) months. The median OS of metastasectomy group and nonmetastasectomy group was 24.6 months $(95 \% \mathrm{CI}, 19.3$ to 30.0 months) and 14.3 months (95\% CI, 10.8 to 17.8 months), respectively. Therefore, patients in the metastasectomy group had a significantly better OS than patients in the nonmetastasectomy group (synchronous ovarian metastasis: $\mathrm{P}<$ 0.001 , Figure 1; metachronous ovarian metastasis: $\mathrm{P}=0.02$, Figure 2).

Based on univariate analysis, metastasectomy, peritoneal carcinomatosis, signet ring cell, ascites, expression of ER- $\beta$ and PR, and serum levels of CA19$9(>37 \mathrm{U} / \mathrm{mL})$ were prognostic factors associated with survival. After adjusting covariates on multivariate analysis, metastasectomy (hazard ration [HR] 0.486; 95\% CI 0.323$0.729 ; P<0.001)$, presence of peritoneal carcinomatosis (HR 1.934; 95\% CI 1.230-3.049; $P=0.004$ ), ER- $\beta$ positivity (HR $0.404 ; 95 \%$ CI $0.251-0.648 ; P<0.001$ ), and PR positivity (HR $0.496 ; 95 \%$ CI $0.301-0.817 ; P<0.001$ ) were independent predictors of OS (Table 3).

Additionally, 63.3\% (57/90) of the patients had R0 resection. Median OS was 30.5 (95\% CI 25.1-35.9) months in the R0 resection group and 12.2 (95\% CI 10.414.0) months in the non-R0 resection groups. Survival was superior in the R0 resection group as compared to the non-R0 resection groups $(P<0.001$; Figure 3$)$.

\section{Correlation between expression of sex hormone receptors, clinicopathologic factors, and survival}

The positive rate of ER- $\beta$ and PR was $44.7 \%$ and $31.6 \%$.However, ER- $\alpha$ showed no positivity in any gastric cancer tissue sample.

The positive/negative expression profiles of representative sex hormone receptors are shown in Figures 4 and 5. Uni- and multivariate analyses showed a positive correlation between expression of ER- $\beta$ and PR and better survival. The average OS in ER- $\beta$-positive and -negative patients was 24.4 (95\% CI 21.5-27.4) months and 12.6 (95\% CI 10.8-14.4) months, respectively $(P<0.001$; Figure 6). Average OS in PR-positive and -negative patients was 23.7 (95\% CI 20.2-27.3) months and 15.4 (95\% CI 13.2$17.5)$ months, respectively $(P<0.001$; Figure 7$)$. These results indicate that expression of ER- $\beta$ or PR are favorably associated with better prognosis in patients with ovarian metastases from a primary gastric cancer.

Furthermore, we evaluated the relationship between the expression of ER- $\beta, P R$, and clinicopathologic factors, and found negative expression of ER- $\beta$ to be significantly associated with peritoneal metastasis, signet-ring cells, and ascites; negative expression of PR was significantly associated with laterality as well as peritoneal metastasis [Table 4]. On multivariate analysis, peritoneal metastasis was significantly associated with negative expression of ER- $\beta$ and PR. In addition, laterality was also significantly associated with negative expression of PR.

\section{Chemotherapy regimens and toxicity}

Paclitaxel and S-1 were the most frequently used chemotherapeutic drugs. Median chemotherapy duration 
Table 1: Clinical characteristics of 93 patients with synchronous Krukenberg tumors

\begin{tabular}{|c|c|c|c|}
\hline Variable & $\begin{array}{c}\text { Metastasectomy plus } \\
\text { chemotherapy } \\
(n=49)\end{array}$ & $\begin{array}{l}\text { Chemotherapy alone } \\
\qquad(n=44)\end{array}$ & $P$-value \\
\hline Median age & $43.1(26-65)$ & $40.9(18-61)$ & 0.290 \\
\hline Tumor size $(\mathrm{cm})$ & $8.70(2-20)$ & $7.33(2.2-18)$ & 0.119 \\
\hline \multicolumn{4}{|l|}{ ECOG performance status } \\
\hline $0-1$ & 47 & 40 & 0.326 \\
\hline 2 & 2 & 4 & \\
\hline \multicolumn{4}{|l|}{ Laterality } \\
\hline Bilateral & 33 & 31 & 0.747 \\
\hline UnilateraI & 16 & 13 & \\
\hline \multicolumn{4}{|l|}{ Peritoneal metastasis } \\
\hline No & 35 & 37 & 0.145 \\
\hline Yes & 14 & 7 & \\
\hline \multicolumn{4}{|l|}{ Signet-ring cells } \\
\hline Positive & 36 & 29 & 0.427 \\
\hline Negative & 13 & 15 & \\
\hline \multicolumn{4}{|l|}{ Differentiation } \\
\hline Well and moderately & 8 & 4 & 0.299 \\
\hline Poorly & 41 & 40 & \\
\hline \multicolumn{4}{|l|}{ Ascites } \\
\hline No & 20 & 11 & 0.106 \\
\hline Yes & 29 & 33 & \\
\hline \multicolumn{4}{|l|}{ ER } \\
\hline Positive & 22 & 14 & 0.196 \\
\hline Negative & 27 & 30 & \\
\hline \multicolumn{4}{|l|}{ PR } \\
\hline Positive & 16 & 8 & 0.111 \\
\hline Negative & 33 & 36 & \\
\hline \multicolumn{4}{|l|}{ Serum CEA (ng/mL) } \\
\hline Normal & 40 & 36 & 0.982 \\
\hline$>5$ & 9 & 8 & \\
\hline \multicolumn{4}{|l|}{ Serum CA19-9 (U/mL) } \\
\hline Normal & 33 & 26 & 0.409 \\
\hline$>39$ & 16 & 18 & \\
\hline \multicolumn{4}{|l|}{ Serum CA125 (U/mL) } \\
\hline Normal & 20 & 14 & 0.368 \\
\hline$>35$ & 29 & 30 & \\
\hline
\end{tabular}


Table 2: Clinical characteristics of 59 patients with metachronous Krukenberg tumors

\begin{tabular}{|c|c|c|c|}
\hline Variable & $\begin{array}{c}\text { Metastasectomy plus } \\
\text { chemotherapy } \\
(n=40)\end{array}$ & $\begin{array}{l}\text { Chemotherapy alone } \\
\qquad(n=19)\end{array}$ & $P$-value \\
\hline Median age & $46.9(31-62)$ & $42.7(20-60)$ & 0.128 \\
\hline Tumor size $(\mathrm{cm})$ & $8.78(4.3-20)$ & $7.73(4.5-16.7)$ & 0.261 \\
\hline \multicolumn{4}{|l|}{ ECOG performance status } \\
\hline $0-1$ & 39 & 17 & 0.190 \\
\hline 2 & 1 & 2 & \\
\hline \multicolumn{4}{|l|}{ Laterality } \\
\hline Bilateral & 32 & 16 & 0.700 \\
\hline UnilateraI & 8 & 3 & \\
\hline \multicolumn{4}{|l|}{ Peritoneal metastasis } \\
\hline No & 29 & 15 & 0.595 \\
\hline Yes & 11 & 4 & \\
\hline \multicolumn{4}{|l|}{ Signet-ring cells } \\
\hline Positive & 31 & 16 & 0.550 \\
\hline Negative & 9 & 3 & \\
\hline \multicolumn{4}{|l|}{ Differentiation } \\
\hline Well and moderately & 6 & 4 & 0.563 \\
\hline Poorly & 34 & 15 & \\
\hline \multicolumn{4}{|l|}{ AJCC stage } \\
\hline $\mathrm{I}-\mathrm{II}$ & 5 & 1 & 0.390 \\
\hline III-IV & 35 & 18 & \\
\hline \multicolumn{4}{|l|}{ Ascites } \\
\hline No & 9 & 5 & 0.748 \\
\hline Yes & 31 & 14 & \\
\hline \multicolumn{4}{|l|}{ ER } \\
\hline Positive & 17 & 8 & 0.977 \\
\hline Negative & 23 & 11 & \\
\hline \multicolumn{4}{|l|}{ PR } \\
\hline Positive & 13 & 6 & \\
\hline Negative & 27 & 13 & 0.944 \\
\hline \multicolumn{4}{|l|}{ Serum CEA (ng/mL) } \\
\hline Normal & 33 & 16 & 0.870 \\
\hline$>5$ & 7 & 3 & \\
\hline \multicolumn{4}{|l|}{ Serum CA19-9 (U/mL) } \\
\hline Normal & 31 & 13 & 0.454 \\
\hline$>39$ & 9 & 6 & \\
\hline \multicolumn{4}{|l|}{ Serum CA125 (U/mL) } \\
\hline Normal & 21 & 7 & 0.260 \\
\hline$>35$ & 19 & 12 & \\
\hline
\end{tabular}


was longer in the metachronous group (4.5 cycles, range 3-8 cycles) as compared to the synchronous group (3.7 cycles, range 1-7 cycles) although the difference was not statistically significant $(P>0.05)$. Drug-related adverse events and grade 3 or 4 toxicity occurred in $67.1 \%$ $(102 / 152)$ and $37.5 \%(57 / 152)$ of patients, respectively. The most common grade 3 or 4 hematological toxic effects were leucopenia/neutropenia(26.3\%) and thrombocytopenia( $7.9 \%$ ); the most common grade 3 or 4 nonhematological toxic effects were elevated serum aspartate aminotransferase levels (4.6\%) and rash $(2.6 \%)$.

\section{Postoperative complications}

Fifteen patients (16.9\%) developed postoperative complications, including anastomotic leakage $(n=5)$, abdominal abscesses $(n=4)$, delayed gastric emptying $(n=4)$, pneumonia $(n=2)$, and bleeding $(n=1)$. Most of these patients were conservatively managed with a successful outcome, but reoperation was necessary in one patient due to intra-abdominal bleeding.

\section{DISCUSSION}

Cancers of the gastrointestinal tract are most likely to generate ovarian metastases in female patients and this is one of the most important causes of treatment failure [11]. The prognosis of patients with gastric cancer and metastasis to the ovaries has been reported to be poorer, as compared with prognosis in other primary gastrointestinal tumors [12]. In the past, chemotherapy was the main treatment for gastric cancer with ovarian metastasis, but the efficiency was disappointing, with a median survival time of 7 to 11 months [13]. Some retrospective study reports in recent years indicated that metastasectomy of Krukenberg tumors could improve prognosis of these patients $[7,8,9]$. However, an optimal therapeutic strategy for Krukenberg tumors from gastric cancer has not been fully established.

$\mathrm{Lu}$ et al. retrospectively reviewed a series of 85 patients diagnosed with advanced gastric cancer together with Krukenberg tumor between 2000 and 2010, and reported a median survival time of 14.1 months in the resection group as compared to 8 months in the nonresection group [7]. In another paper, Cho et al. reported that OS differed significantly between patients undergoing metastasectomy plus chemotherapy and those undergoing chemotherapy alone (18.0 months vs. 8.0 months in patients with stage IV gastric cancer; 19.0 months vs. 9.0 months in patients with recurrent Krukenberg tumors).Nevertheless, the imbalance between the two groups should cast some doubts on the significance of the findings [8].

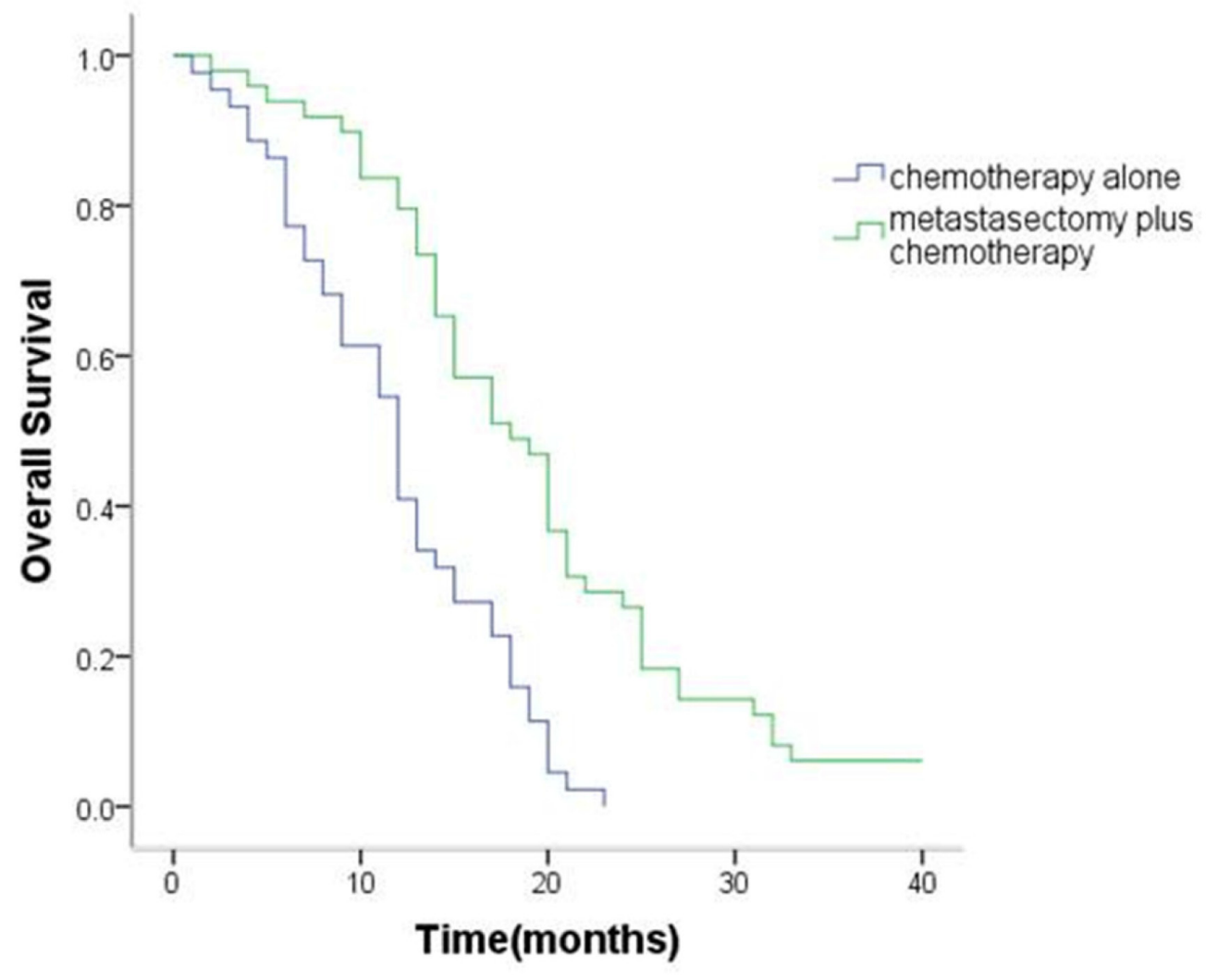

Figure 1: Kaplan-Meier analysis of overall survival in patients with or without metastasectomy of synchronous Krukenberg tumors. 
In the present study, we retrospectively analyzed outcomes of 152 patients with synchronous and metachronous ovarian metastasis. Metastasectomy, relative to non-metastasectomy treatment, resulted in significantly better OS in patients with synchronous (19.0 months vs. 13.5 months; $P<0.001)$ and metachronous (24.6 months vs. 14.3 months; $P=$ 0.02 ) ovarian metastasis. The present series included a large study sample, with similar clinicopathologic characteristics for both study groups. Our results are in accordance with those reported in the earlier literature, therefore, we can conclude that metastasectomy should be recommended in patients with ovarian metastasis.

Analysis of prognostic factors can, moreover, facilitate identification of patients most likely to benefit from treatment. Metastasectomy, peritoneal carcinomatosis, and expression of ER- $\beta$ and PR were independent predictors of OS. Krukenberg tumors are often associated with different degrees of peritoneal metastasis, which frequently induces ascites, intestinal obstruction, or hydronephrosis and seriously impair patient quality of life [14]. There is currently no standard treatment for ovarian metastasis when it presents with peritoneal metastases from gastric cancer, however, cytoreductive surgery in combination with chemotherapy could offer a survival advantage for these patients [15, 16]. In our study, limited peritoneal metastases were resected in a metastasectomy, and this was followed by systemic chemotherapy. Subgroup analysis showed that the survival of the $\mathrm{R} 0$ resection group was superior to that of the non-R0 resection group. Therefore, surgical resection without gross residual disease may improve prognosis in patients with Krukenberg tumors. Chemotherapy is another main treatment for these patients, and improves quality of life and OS [17]. Several chemotherapeutic agents are effective against Krukenberg tumors with peritoneal metastasis, including fluoropyrimidine, platinum, taxanes, and epirubicin, either alone or in combination [18, 19]. A European study reported encouraging results from the use of hyperthermic intraperitoneal chemotherapy (HIPEC) [5], but these need to be validated in future research.

Some studies have reported a correlation between expression of sex hormone receptors and the incidence and progression of gastric cancer [20]. It has been suggested that sex hormones play critical protective roles in female patients with gastric cancer [21, 22]. Recently, a meta-analysis suggested that longer exposure to the effects of estrogen may potentially decrease the risk of gastric cancer [23]. However, the presence and

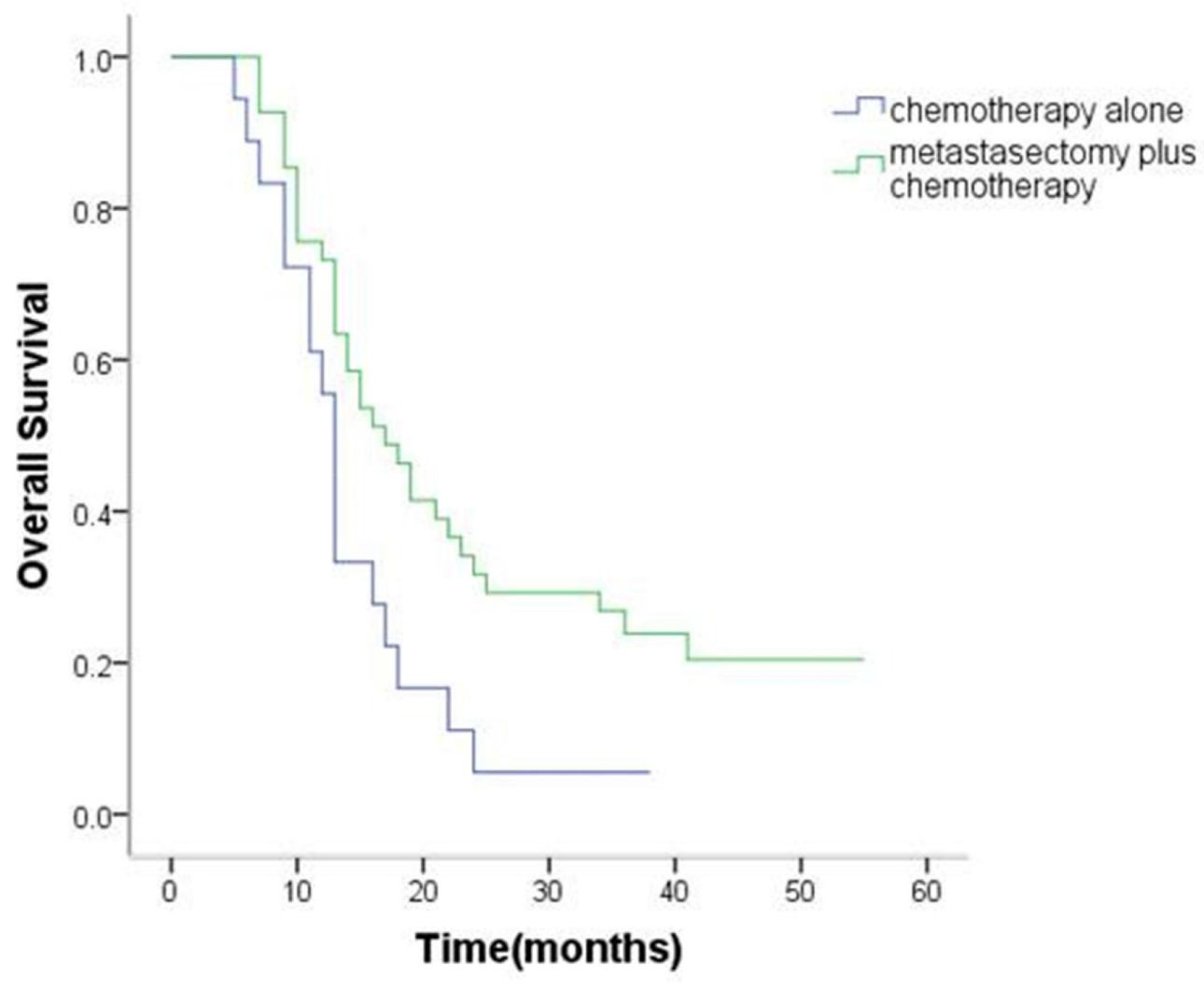

Figure 2: Kaplan-Meier analysis of overall survival in patients with or without metastasectomy of metachronous Krukenberg tumors. 
Table 3: Univariate and multivariate analysis of overall survival

\begin{tabular}{|c|c|c|c|c|c|c|}
\hline \multirow{2}{*}{\multicolumn{2}{|c|}{ Variable }} & \multicolumn{2}{|c|}{ Univariate } & \multicolumn{3}{|c|}{ Multivariate } \\
\hline & & HR (95\% CI) & $P$-value & & HR (95\% CI) & $P$-value \\
\hline \multicolumn{2}{|l|}{ Age $(\geq 50$ years $)$} & $0.712(0.445-1.139)$ & 0.156 & & - & - \\
\hline \multicolumn{2}{|c|}{ Size of tumor $(<5 \mathrm{~cm})$} & $0.736(0.491-1.104)$ & 0.139 & & - & - \\
\hline \multicolumn{2}{|c|}{ Bilateral ovarian metastases } & $0.775(0.515-1.165)$ & 0.220 & & - & - \\
\hline \multicolumn{2}{|l|}{ Metastasectomy } & $0.467(0.318-0.685)$ & $<0.001$ & & $0.486(0.323-0.729)$ & $<0.001$ \\
\hline \multicolumn{2}{|c|}{ Peritoneal carcinomatosis } & $2.359(1.585-3.512)$ & $<0.001$ & & $1.934(1.230-3.049)$ & 0.004 \\
\hline \multicolumn{2}{|l|}{ Signet-ring cells } & $1.871(1.272-2.751)$ & 0.001 & & $1.183(0.778-1.802)$ & 0.430 \\
\hline \multicolumn{2}{|l|}{ Ascites } & $1.968(1.269-3.051)$ & 0.002 & & $1.450(0.914-2.294)$ & 0.114 \\
\hline \multicolumn{2}{|l|}{ Gastrectomy } & $0.761(0.520-1.114)$ & 0.160 & & - & - \\
\hline \multicolumn{2}{|l|}{ ER positive } & $0.254(0.166-0.389)$ & $<0.001$ & & $0.404(0.251-0.648)$ & $<0.001$ \\
\hline \multicolumn{2}{|l|}{ PR positive } & $0.376(0.238-0.596)$ & $<0.001$ & & $0.496(0.301-0.817)$ & $<0.001$ \\
\hline \multicolumn{2}{|l|}{ CA125 } & $1.427(0.939-2.170)$ & 0.096 & & - & - \\
\hline \multicolumn{2}{|l|}{ CEA } & $1.369(0.873-2.146)$ & 0.171 & & - & - \\
\hline \multicolumn{2}{|l|}{ CA199 } & $1.628(1.108-2.393)$ & 0.013 & & $1.447(0.963-2.179)$ & 0.075 \\
\hline
\end{tabular}

Figure 3: Kaplan-Meier analysis of overall survival in patients with or without R0 resection. 
role of sex hormone receptors in Krukenberg tumors were undetermined. We evaluated the expression of ER-a, ER- $\beta$, and PR in gastric cancer specimens, and found that only ER- $\beta$ and PR were detected. Our data showed that positive expression of ER- $\beta$ or PR were favorably associated with better prognosis of gastric cancer patients with ovarian metastases. From our results and those reported in the related literature, ER and PR play an important role in the etiopathogenesis of Krukenberg tumors. However, some issues remain to be resolved. It is unclear whether targeted therapy of sex hormone receptors can lower the incidence of ovarian metastasis from ER- or PR-positive gastric cancer. Moreover, the possibility of ER or PR expression being used as a reliable indicator of prophylactic ovariectomy is worth exploring. Thus, future studies investigating the significance of ER or PR expression in carcinogenesis and tumor progression of gastric cancer and Krukenberg tumor are necessary.

Our study had certain limitations because it was a retrospective analysis, and several confounding factors may have influenced our findings. However, the fact that it reports results from a large series of patients and presents the first evaluation of the role of sex hormone
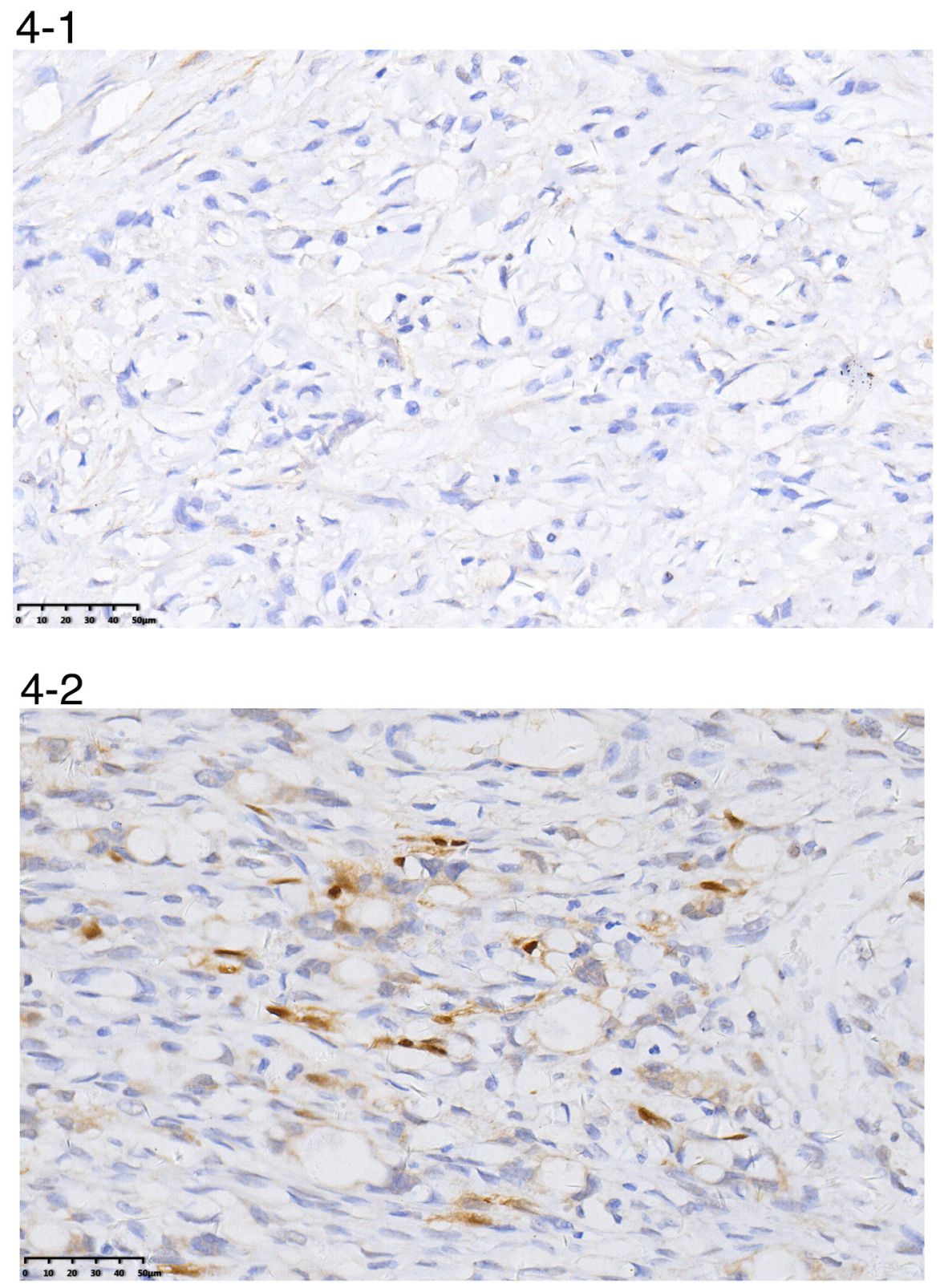

Figure 4: A representative image of negative (4-1) or positive (4-2) ER- $\beta$ expression. 
receptors in Krukenberg tumors from gastric cancer. The results are expected to provide useful information for formulating treatment strategies for those patients.

The present study demonstrated that the metastasectomy in combination with chemotherapy was associated with survival benefits in patients with synchronous or metachronous Krukenberg tumors from gastric cancer. Metastasectomy, peritoneal carcinomatosis, and ER- $\beta$ and PR expression status were prognostic factors for survival. Well-designed prospective studies are needed to confirm these results, and will be important in developing optimal treatment strategies for Krukenberg tumors of gastric origin.
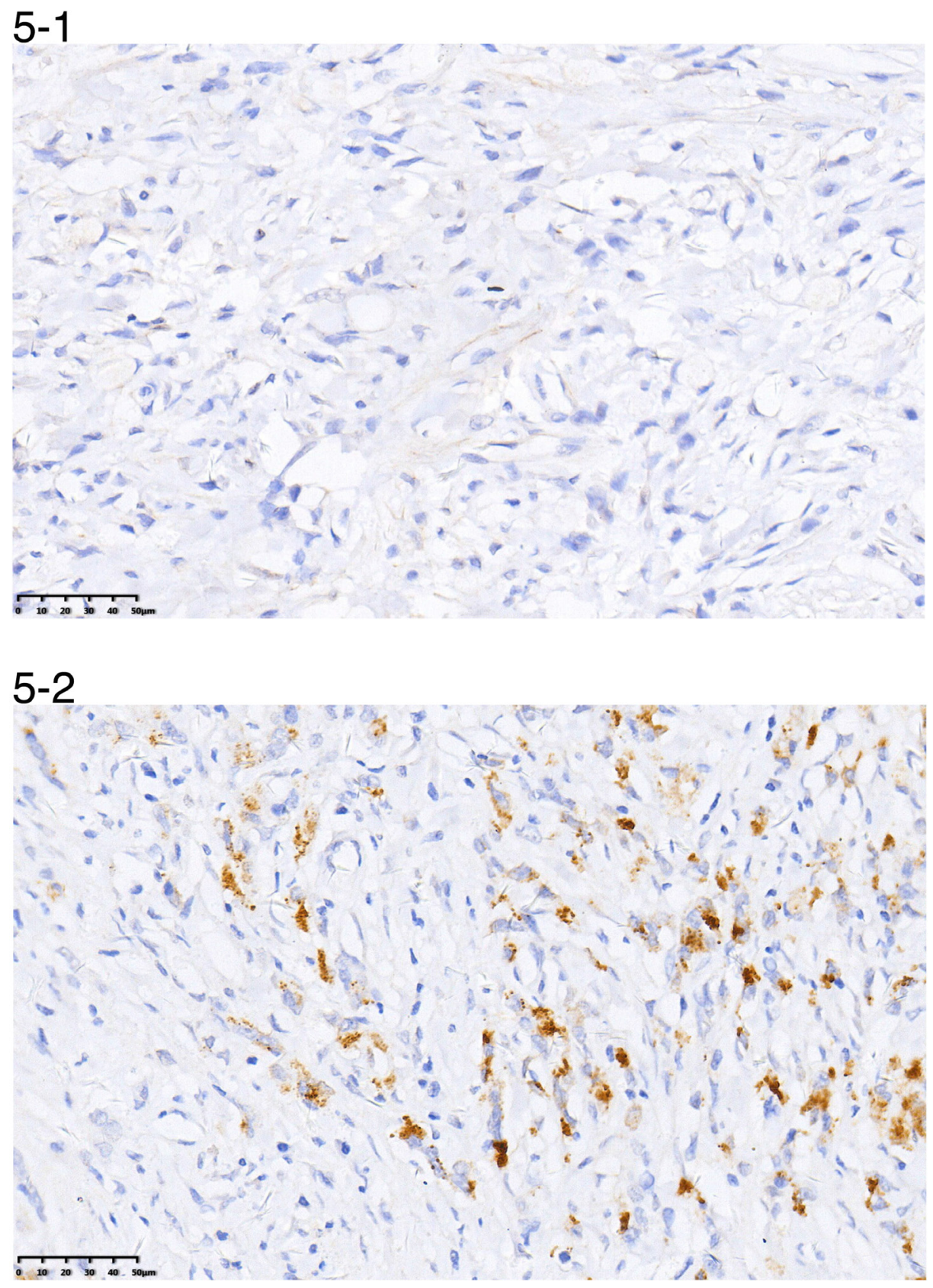

Figure 5: A representative image of negative (5-1) or positive (5-2) PR expression. 


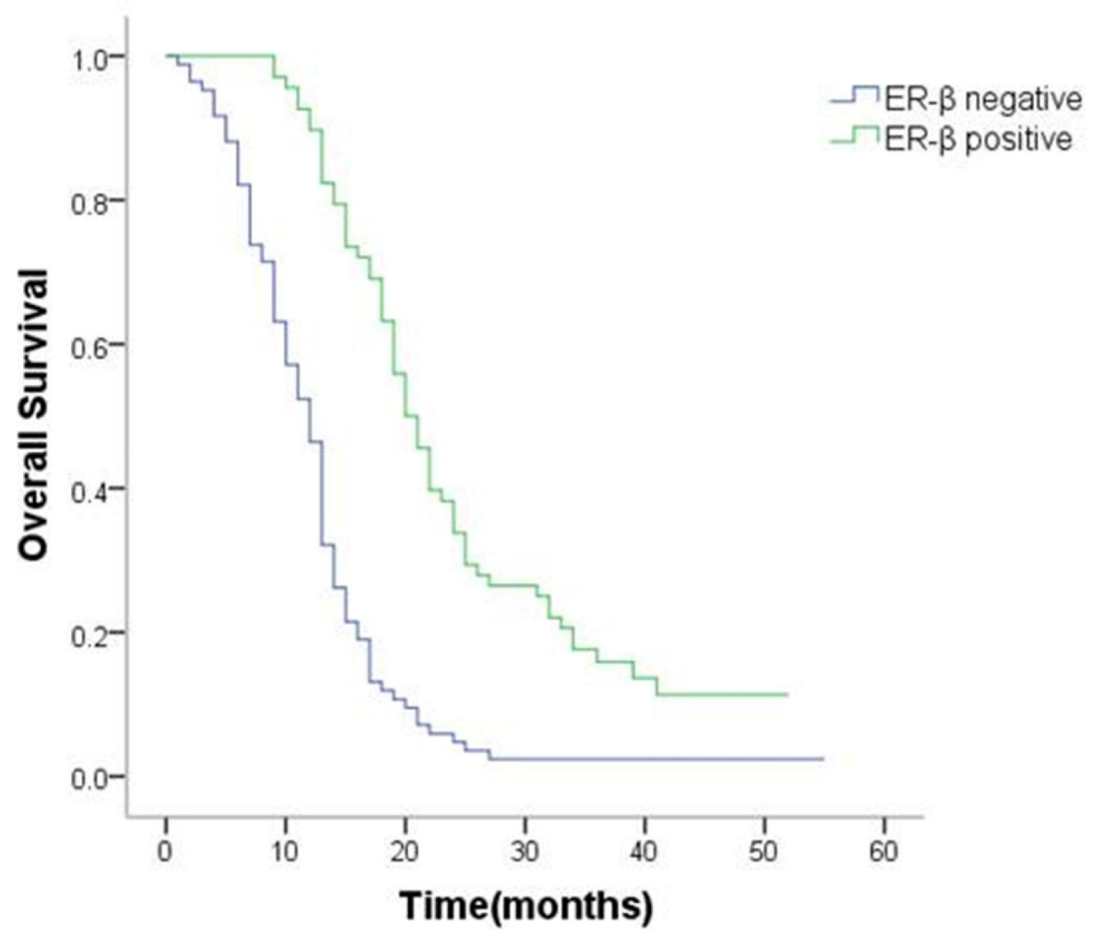

Figure 6: Survival curves of patients with positive or negative expression of ER-ß.

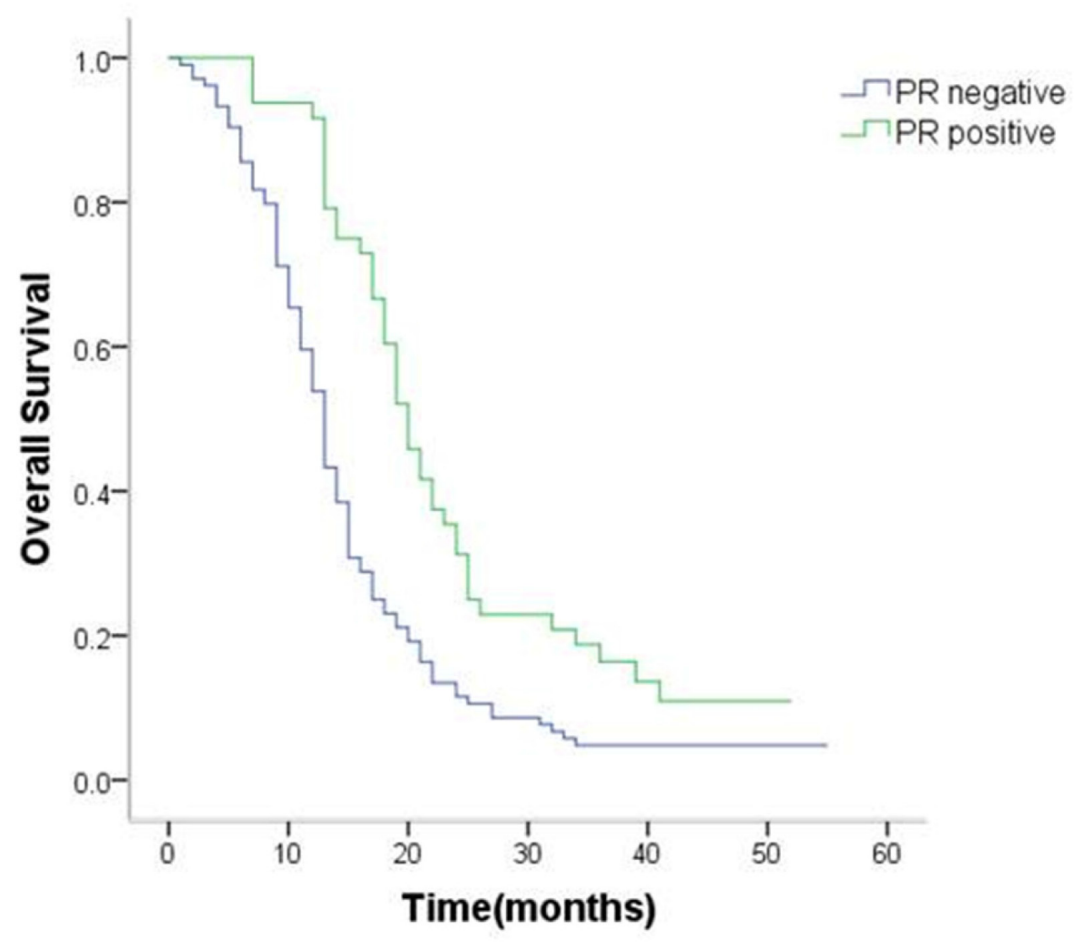

Figure 7: Survival curves of patients with positive or negative expression of PR. 
Table 4: Correlation between ER- $\beta$ and PR expressions and clinicopathologic characteristics.

\begin{tabular}{|c|c|c|c|c|c|c|}
\hline & \multicolumn{3}{|c|}{ ER- $\boldsymbol{\beta}$} & \multicolumn{3}{|c|}{ PR } \\
\hline & $(+)$ & $(-)$ & $P$ & $(+)$ & $(-)$ & $P$ \\
\hline \multicolumn{7}{|l|}{ Age (years) } \\
\hline$\geq 50$ & 16 & 22 & 0.706 & 16 & 22 & 0.107 \\
\hline$<50$ & 52 & 62 & & 32 & 82 & \\
\hline \multicolumn{7}{|l|}{ Tumor size (cm) } \\
\hline$\geq 5$ & 48 & 58 & 0.837 & 38 & 68 & 0.086 \\
\hline$<5$ & 20 & 26 & & 10 & 36 & \\
\hline \multicolumn{7}{|l|}{ Laterality } \\
\hline Bilateral & 50 & 57 & 0.446 & 41 & 67 & 0.008 \\
\hline UnilateraI & 18 & 27 & & 7 & 37 & \\
\hline \multicolumn{7}{|c|}{ Peritoneal metastasis } \\
\hline No & 57 & 44 & $<0.001$ & 40 & 61 & 0.003 \\
\hline Yes & 11 & 40 & & 8 & 43 & \\
\hline \multicolumn{7}{|l|}{ Signet-ring cells } \\
\hline Positive & 26 & 46 & 0.042 & 21 & 51 & 0.544 \\
\hline Negative & 42 & 38 & & 27 & 53 & \\
\hline \multicolumn{7}{|l|}{ Ascites } \\
\hline No & 30 & 23 & 0.031 & 19 & 34 & 0.407 \\
\hline Yes & 38 & 61 & & 29 & 70 & \\
\hline \multicolumn{7}{|c|}{ Ovarian metastases } \\
\hline Synchronous & 41 & 52 & 0.839 & 28 & 65 & 0.624 \\
\hline Metachronous & 27 & 32 & & 20 & 39 & \\
\hline \multicolumn{7}{|c|}{ Serum CEA (ng/mL) } \\
\hline Normal & 58 & 61 & 0.059 & 38 & 81 & 0.859 \\
\hline$>5$ & 10 & 23 & & 10 & 23 & \\
\hline \multicolumn{7}{|c|}{ Serum CA19-9 (U/mL) } \\
\hline Normal & 43 & 53 & 0.986 & 31 & 65 & 0.805 \\
\hline$>39$ & 25 & 31 & & 17 & 39 & \\
\hline \multicolumn{7}{|c|}{ Serum CA125 (U/mL) } \\
\hline Normal & 27 & 25 & 0.199 & 14 & 38 & 0.373 \\
\hline$>35$ & 41 & 59 & & 34 & 66 & \\
\hline
\end{tabular}

\section{MATERIALS AND METHODS}

\section{Patients}

From January 2005 to December 2014, 4381 female gastric cancer patients were admitted and treated at the Zhejiang Cancer Hospital. Among these patients, 152 with krukenberg tumor detected by the imaging studies or by pathological evaluation of the metastasectomy specimens were retrospectively reviewed. Patient demographics, radiological details, surgical data, pathological features, and survival were collected and analyzed. Outpatient records combined with telephone interviews were used for follow-up. All the patients suspected of having Krukenberg tumor underwent imaging studies to identify the extent of disease and resectability. The patients included in this 
study were initially regarded as having resectable diseases. Therefore, a resection was only performed in case of Krukenberg tumor without or with limited peritoneal dissemination. At the completion of surgery, the residual disease state of each patient was recorded according to the presence or absence of gross residual disease, which was classified as negative resection margins (R0), microscopic tumor infiltration (R1), and macroscopic residual tumor (R2).

Overall, 93 patients were initially diagnosed as synchronous metastasis and 59 as metachronous metastasis after they underwent curative resection of gastric cancer. Patients were divided into two groups according to treatment modality: the metastasectomy group( received both chemotherapy and metastasectomy for Krukenberg tumor) and the non-metastasectomy group(received chemotherapy alone). Overall survival (OS) of patients with synchronous ovarian metastasiswas defined as duration from the date of pathologic diagnosis of gastric cancer to the date of death or last follow-up. OS of patients with metachronous ovarian metastasis was defined as duration from the date of radiologic diagnosis of Krukenberg tumor to the date of death or last follow-up. For both groups, December 31, 2016 was the cutoff date for OS.

\section{Detection of sex hormone receptors in gastric cancer specimens}

The expression of estrogen receptor (ER)- $\alpha$, ER- $\beta$, and progesterone receptor (PR) in gastric cancer, which may potentially be related to the ovarian metastasis, was detected by immunohistochemistry (IHC) using the Novolink Polymer Detection System as described previously [10]. The following primary antibodies were used: ER- $\alpha$ (ab37438, dilution 1:200; Abcam, Cambridge, UK), ER- $\beta$ (ab288, dilution 1:100; Abcam), and PR (ab16661, dilution 1:100; Abcam). All specimens were independently evaluated by two pathologists who were blinded to study grouping. Samples where more than $10 \%$ of the tumor cells were stained were regarded as positive.

\section{Ethics statement}

The study was approved by the institutional ethics review board of Zhejiang Cancer Hospital. Written informed consent was obtained from all study participants. All study procedures were undertaken in accordance with the principles of the Declaration of Helsinki and relevant human research policies in China.

\section{Statistical analysis}

For continuous variables, two-tailed Student $t$ tests were used to compare demographic and clinical characteristics between the study groups. For discrete variables, a chi-square test was used. Kaplan-Meier analysis was used to calculate survival, and log-rank test was performed to compare OS between treatment groups. Independent prognostic factors were determined by multivariate analysis using the Cox proportional hazards model. All statistical analyses were conducted on SPSS 19.0 for Windows (IBM Corporation, Armonk, NY, USA). A $P$ value less than 0.05 was considered indicative of statistical significance.

\section{Author contributions}

Pengfei Yu, Litao Yang, Gaiguo Dai, Jieer Ying, Yian Du: Concept and design of study; Ling Huang, Guoping Cheng,:analysis and interpretation of data; Pengfei Yu, Ling Huang and Yian Du, Drafting the article; Pengfei Yu and Yian Du :Final approval of the version to be published.

\section{ACKNOWLEDGMENTS}

We express our appreciation to Professor Ahmed O. Kaseb (Gastrointestinal Medical Oncology, M. D. Anderson Cancer Center) who has offered many valuable comments and suggestions.

\section{CONFLICTS OF INTEREST}

The authors declare no competing interests.

\section{FUNDING}

This study was supported by Natural Science Foundation of Zhejiang Province of China (No. LY14H160007) and "1022 Talent Plan" of Zhejiang Cancer Hospital.

\section{REFERENCES}

1. Torre LA, Bray F, Siegel RL, Ferlay J, Lortet-Tieulent J, Jemal A. Global cancer statistics, 2012. CA Cancer J Clin. 2015;65:87-108.

2. Matsushita H, Watanabe $\mathrm{K}$, Wakatsuki A. Metastatic gastric cancer to the female genital tract. Mol Clin Oncol. 2016;5:495-9.

3. Wang J, Shi YK, Wu LY, Wang JW, Yang S, Yang JL, Zhang HZ, Liu SM. Prognostic factors for ovarian metastases from primary gastric cancer. Int $\mathrm{J}$ Gynecol Cancer. 2008;18:825-32.

4. Kim HK, Heo DS, Bang YJ, Kim NK. Prognostic factors of Krukenberg's tumor. Gynecol Oncol. 2001;82:105-9.

5. Rosa F, Marrelli D, Morgagni P, Cipollari C, Vittimberga G, Framarini M, Cozzaglio L, Pedrazzani C, Berardi S, Baiocchi GL, Roviello F, Portolani N, de Manzoni G, et al. Krukenberg tumors of gastric origin: the rationale 
of surgical resection and perioperative treatments in a multicenter western experience. World J Surg. 2016;40:921-8.

6. Brieau B, Auzolle C, Pozet A, Tougeron D, Bouché O, Soibinet P, Coriat R, Prieux C, Lecomte T, Goujon G, Marthey L, Rougier P, Bonnetain F, et al. Efficacy of modern chemotherapy and prognostic factors in patients with ovarian metastases from gastric cancer: a retrospective AGEO multicentre study. Dig Liver Dis. 2016;48:441-5.

7. Lu LC, Shao YY, Hsu CH, Hsu C, Cheng WF, Lin YL, Cheng AL, Yeh KH. Metastasectomy of Krukenberg tumors may be associated with survival benefits in patients with metastatic gastric cancer. Anticancer Res. 2012;32:3397-401.

8. Cho JH, Lim JY, Choi AR, Choi SM, Kim JW, Choi SH, Cho JY. Comparison of surgery plus chemotherapy and palliative chemotherapy alone for advanced gastric cancer with Krukenberg tumor. Cancer Res Treat. 2015;47:697-705.

9. Peng W, Hua RX, Jiang R, Ren C, Jia YN, Li J, Guo WJ. Surgical treatment for patients with Krukenberg tumor of stomach origin: clinical outcome and prognostic factors analysis. PLoS One. 2013;8:e68227.

10. Kim HW, Kim JH, Lim BJ, Kim H, Kim H, Park JJ, Youn YH, Park H, Noh SH, Kim JW, Choi SH. Sex disparity in gastric cancer: female sex is a poor prognostic factor for advanced gastric cancer. Ann Surg Oncol. 2016;23:4344-51.

11. Turan T, Aykan B, Koc S, Boran N, Tulunay G, Karacay O, Erdogan Z, Kose F. Analysis of metastatic ovarian tumors from extragenital primary sites. Tumori. 2006;92:491-5.

12. Ayhan A, Guvenal T, Salman MC, Ozyuncu O, Sakinci M, Basaran M. The role of cytoreductive surgery in nongenital cancers metastatic to the ovaries. Gynecol Oncol. 2005;98:235-41.

13. Kiyokawa T, Young RH, Scully RE. Krukenberg tumors of the ovary: a clinicopathologic analysis of 120 cases with emphasis on their variable pathologic manifestations. Am J Surg Pathol. 2006;30:277-99.
14. Kobayashi O, Sugiyama Y, Cho H, Tsuburaya A, Sairenji M, Motohashi H, Yoshikawa T. Clinical and pathological study of gastric cancer with ovarian metastasis. Int J Clin Oncol. 2003;8:67-71.

15. Feng Q, Pei W, Zheng ZX, Bi JJ, Yuan XH. Clinicopathologic characteristics and prognostic factors of 63 gastric cancer patients with metachronous ovarian metastasis. Cancer Biol Med. 2013;10:86-91.

16. Jun SY, Park JK. Metachronous ovarian metastases following resection of the primary gastric cancer. J Gastric Cancer. 2011;11:31-7.

17. Wagner AD, Grothe W, Haerting J, Kleber G, Grothey A, Fleig WE. Chemotherapy in advanced gastric cancer: a systematic review and meta-analysis based on aggregate data. J Clin Oncol. 2006;24:2903-9.

18. Waddell T, Verheij M, Allum W, Cunningham D, Cervantes A, Arnold D. Gastric cancer: ESMO-ESSO-ESTRO Clinical Practice Guidelines for diagnosis, treatment and follow-up. Ann Oncol. 2013;24:vi57-63.

19. Wagner AD, Unverzagt S, Grothe W, Kleber G, Grothey A, Haerting J, Fleig WE. Chemotherapy for advanced gastric cancer. Cochrane Database Syst Rev. 2010;(3):CD004064.

20. Wei S, Said-Al-Naief N, Hameed O. Estrogen and progesterone receptor expression is not always specific for mammary and gynecologic carcinomas: a tissue microarray and pooled literature review study. Appl Immunohistochem Mol Morphol. 2009;17:393-402.

21. Chandanos E, Lagergren J. Oestrogen and the enigmatic male predominance of gastric cancer. Eur $\mathrm{J}$ Cancer. 2008;44:2397-403.

22. Freedman ND, Ahn J, Hou L, Lissowska J, Zatonski W, Yeager M, Chanock SJ, Chow WH, Abnet CC. Polymorphisms in estrogen- and androgen-metabolizing genes and the risk of gastric cancer. Carcinogenesis. 2009;30:71-7.

23. Camargo MC, Goto Y, Zabaleta J, Morgan DR, Correa P, Rabkin CS. Sex hormones, hormonal interventions, and gastric cancer risk: a meta-analysis. Cancer Epidemiol Biomarkers Prev. 2012;21:20-38. 